\title{
Measuring spent fuel assembly multiplication in borated water with a passive neutron albedo reactivity instrument
}

\section{Tobin, Stephen J.}

2018-07-21

Tobin , S J , Peura , P , Bélanger-Champagne , C , Moring , M , Dendooven , P \& Honkamaa , T 2018 , ' Measuring spent fuel assembly multiplication in borated water with a passive neutron albedo reactivity instrument ' , Nuclear Instruments \& Methods in Physics Research. Section A: Accelerators, Spectrometers, Detectors, and Associated Equipment, vol. 897 , pp. 32-37 . https://doi.org/10.1016/j.nima.2018.04.044

http://hdl.handle.net/10138/314628

https://doi.org/10.1016/j.nima.2018.04.044

cc_by_nc_nd

publishedVersion

Downloaded from Helda, University of Helsinki institutional repository.

This is an electronic reprint of the original article.

This reprint may differ from the original in pagination and typographic detail.

Please cite the original version. 


\section{Accepted Manuscript}

Measuring spent fuel assembly multiplication in borated water with a passive neutron albedo reactivity instrument

Stephen J. Tobin, Pauli Peura, Camille Bélanger-Champagne, Mikael Moring, Peter Dendooven, Tapani Honkamaa

PII: $\quad$ S0168-9002(18)30551-5

DOI: $\quad$ https://doi.org/10.1016/j.nima.2018.04.044

Reference: $\quad$ NIMA 60764

To appear in: $\quad$ Nuclear Inst. and Methods in Physics Research, A

Received date: 17 January 2018

Revised date: 15 April 2018

Accepted date : 22 April 2018

Please cite this article as: S.J. Tobin, P. Peura, C. Bélanger-Champagne, M. Moring, P. Dendooven, T. Honkamaa, Measuring spent fuel assembly multiplication in borated water with a passive neutron albedo reactivity instrument, Nuclear Inst. and Methods in Physics Research, A (2018), https://doi.org/10.1016/j.nima.2018.04.044

This is a PDF file of an unedited manuscript that has been accepted for publication. As a service to our customers we are providing this early version of the manuscript. The manuscript will undergo copyediting, typesetting, and review of the resulting proof before it is published in its final form. Please note that during the production process errors may be discovered which could affect the content, and all legal disclaimers that apply to the journal pertain. 
Measuring Spent Fuel Assembly Multiplication in Borated Water with a Passive Neutron Albedo Reactivity Instrument

Stephen J. Tobin ${ }^{1}$, Pauli Peura ${ }^{2}$, Camille Bélanger-Champagne ${ }^{2}$, Mikael Moring ${ }^{3}$, Peter Dendooven ${ }^{2}$, Tapani Honkamaa ${ }^{3}$

${ }^{1}$ Encapsulation Nondestructive Assay Services, Los Alamos, NM 87544, USA.

${ }^{2}$ Helsinki Institute of Physics, FI-00014 University of Helsinki, Finland.

${ }^{3}$ Radiation and Nuclear Safety Authority - STUK, 00881 Helsinki, Finland.

\section{ABSTRACT}

The performance of a passive neutron albedo reactivity (PNAR) instrument to measure neutron multiplication of spent nuclear fuel in borated water is investigated as part of an integrated non-destructive assay safeguards system. To measure the PNAR Ratio, which is proportional to the neutron multiplication, the total neutron count rate is measured in high- and low-multiplying environments by the PNAR instrument. The integrated system also contains a load cell and a passive gamma emission tomograph, and as such meets all the recommendations of the IAEA's recent ASTOR Experts Group report. A virtual spent fuel library for VVER-440 fuel was used in conjunction with MCNP simulations of the PNAR instrument to estimate the measurement uncertainties from (1) variation in the water boron content, (2) assembly positioning in the detector and (3) counting statistics. The estimated aggregate measurement uncertainty on the PNAR Ratio measurement is 0.008 , to put this uncertainty in context, the difference in the PNAR Ratio between a fully irradiated assembly and this same assembly when fissile isotopes only absorb neutrons, but do not emit neutrons, is 0.106 , a 13-sigma effect. The 1-sigma variation of 0.008 in the PNAR Ratio is estimated to correspond to a $3.2 \mathrm{GWd} / \mathrm{tU}$ change in assembly burnup.

KEY WORDS: Spent fuel encapsulation, Non-destructive Assay

\section{INTRODUCTION}

The Finnish Radiation and Safety Authority (STUK), in order to implement the recommendation of the International Atomic Energy Agency (IAEA) assembled NDA experts outlined in the "Application of Safeguards to Geological Repositories (ASTOR) Report on Technologies Potentially Useful for Safeguarding Geological Repositories," [1] funded research to conceptually design two integrated nondestructive assay (NDA) systems; one system to measure boiling water reactor (BWR) fuel and one to measure VVER-440 fuel. The integrated instruments each have three parts, a Passive Gamma Emission Tomography (PGET) instrument [1, 2, 3, 4], a Passive Neutron Albedo Reactivity (PNAR) instrument [1, 5, 6, 7] and a load cell that will measure the assembly weight. This study will focus on the PNAR instrument, which supports several of the recommended characteristics outlined for 
the NDA system by the ASTOR experts. Among those characteristics, PNAR has the unique role, in the integrated system, of measuring the assembly's neutron multiplication. Although the ASTOR participants were organized by the IAEA, their recommendations are not IAEA policy; the inclusion of multiplication as a metric is novel. Multiplication was included because it is a direct indication of the presence of fissile material.

In Finland there will be two measurement locations. The BWR fuel will be measured in fresh water while the VVER-440 fuel, as with most pressurized water reactor spent fuel pools, will be measured in borated water. The task of measuring the assembly's neutron multiplication in borated water reduces the sensitivity of the instrument and increases the uncertainty. The current study quantifies both the anticipated sensitivity and uncertainty of a conceptual PNAR instrument designed to measure VVER-440 fuel in borated water.

\section{PASSIVE NEUTRON ALBEDO REACTIVITY PHYSICS}

The PNAR concept involves the comparison of the neutron count rate of an object when that object is measured in two different setups. One setup is designed to enhance neutron multiplication while the other setup is designed to suppress it. As implemented in Finland, the high multiplying section is produced by the assembly in water, while the low multiplying section is created by putting $1 \mathrm{~mm}$ of Cd as close as possible to the fuel while it remains in the pool. As the result of criticality safety regulations, the water in pool containing VVER-440 fuel is borated, while the pool containing BWR fuel is fresh. Cd was selected for the low multiplying section due to its extremely large absorption cross-section for all neutron energies below $\sim 0.5 \mathrm{eV}$. The PNAR signature, the PNAR Ratio, is calculated by dividing the count rate measured in the high multiplying section by the count rate measured in the low multiplying section.

The PNAR implementation in Finland, an implementation that combines (a) a ${ }^{3} \mathrm{He}$ detector tube and polyethylene (PE) surrounded by $\mathrm{Cd}$ and (b) a low multiplying section produced with a Cd-liner, lends itself to a conceptual discussion of the PNAR physics. The only significant difference in the measured count rate for a section of fuel measured in both the high and low multiplying sections, is the counts resulting from the multiplication caused by the neutrons that are absorbed in the Cd-liner. The contribution from neutrons not absorbed in the Cd-liner, are in both the numerator and denominator of the PNAR Ratio. In isolation, these high-energy neutrons that are unaffected by the Cd-liner create a PNAR Ratio of 1.0; any deviation from 1.0 is due to counts produced by chain reactions initiated by neutrons that are absorbed by the Cd-liner. Because the PNAR signal is produced by the neutrons returning into the fuel with an energy below the Cd-cutoff energy of $\sim 0.5 \mathrm{eV}$, the PNAR technique is sometimes described as interrogating the fuel with low energy neutrons from the location of the Cd-liner. 
The PNAR conceptual design is part of an integrated NDA system that needs to meet the safeguards and safety needs of Finland in the context of VVER-440 spent fuel encapsulation and geological disposal. In Figure 1, a vertical cross-cut of the VVER440 PNAR detector module is illustrated. In Figure 2 a horizontal cross-cut illustrates that there are three detectors around the assembly at one axial location. Below the three detector modules illustrated at one axial level are three more detectors which are rotated around the fuel assembly by 60 degrees. The two levels are separated by $\sim 0.1$ $\mathrm{m}$. In Figure 3, the full $74 \mathrm{~cm}$ vertical extent of the detector is evident as well as the vertical separation between the two detector layers as one detector from each layer is shown. This number of detectors was selected to improve simulation statistics as well as to enable research into how the number of detectors impacts the sensitivity of the instrument to assembly location in the detector. The final deployment is expected to have three detectors unless there is some need for redundant instruments.

Several aspects of the PNAR design are listed here:

- The ${ }^{3} \mathrm{He}$ in the neutron detector has a $0.1 \mathrm{~m}$ active length, $17.4 \mathrm{~mm}$ or $3 / 4^{\text {th }}$ inch diameter, 6 atm pressure, and is surrounded by a cylinder of $\mathrm{PE}$ that has a diameter of $58 \mathrm{~mm}$.

- The ${ }^{3} \mathrm{He}$ tube and cylindrical PE are surrounded by a layer of cadmium so that, as a unit, the detector module detects primarily epithermal and fast neutrons incident upon it.

- The layer of lead is $46 \mathrm{~mm}$ thick at the thickest point in Figure1.

Location of Figure 1

Location of Figure 2

Location of Figure 3

The Cd-liner located close to the fuel, the full $0.74 \mathrm{~m}$ length of which is indicated in Figure 3, is the core hardware part needed to implement the PNAR concept. This Cdliner, in the Finnish implementation of PNAR, will be mobile. For the low multiplication part of the PNAR measurement, it will be located as illustrated and for the high multiplying part it will be moved below the PE slab.

The PE slab located outside the detector modules is there for two primary reasons: (a) to raise the neutron multiplication of an assembly inside the detector when the Cdliner is not present and (b) to reduce the uncertainty in the neutron count rate resulting from the variation in the boron content of the water 
To assess the capability of the PNAR detector customized for VVER-440 fuel, the PNAR Ratio was simulated and calculated using 12 assemblies that span a range of initial enrichment (3, 4 and 5 wt.\%) and burnup (15, 30, 45 and $60 \mathrm{GWd} / \mathrm{tU})$ for a cooling time of 20 -years. The cooling time of 20 years was selected because the Finnish repository expects to accept fuel that cooled between 20 and 60 years; additionally, as noted on page 48 of [1], the multiplication of typical assemblies is expected to change by less than $10 \%$ over this time range. Each assembly was chosen to have a uniform isotopic content that matches the average content for an assembly of the given characteristics. The Monte Carlo N-Particle Code Version 6 (MCNP6 ${ }^{\mathrm{TM}}$ ) [8] with $0.80 \mathrm{c}$ cross sections [9] was used for the PNAR simulations. The isotopic mixture of the various assemblies was produced by the Monteburns code [10] as part of the Next Generation Safeguards Initiative [11, 12]. As many irradiation codes accurately simulate the neutron transport relevant isotopic content of an irradiated assembly, several different codes could be used. Per input from the Finnish facility operator, the boron content of the water was taken to be $14 \mathrm{~g}$ of boric acid per $\mathrm{kg}$ of water.

In Figure 4, the calculated PNAR Ratio for 12 different assemblies in borated water is illustrated. All the data points are for fuel with a 20 years cooling time. The PNAR Ratio values for all the data points with ratios above 1.1 were simulated in the standard manner, meaning that all neutrons, and subsequent reactions that these neutrons might cause, were followed until the neutrons were either absorbed or left the exterior boundaries of the simulation. This boundary was defined as a cuboid, 0.4 $\mathrm{m}$ on two sides that extended 1.6 meters in the vertical direction. Any nuclear reactions that produced additional neutrons, such as induced fission, were followed through to fruition.

\section{Location of Figures 4.}

For the three assemblies that were irradiated to the level at which assemblies are generally removed from a commercial reactor $(3 \mathrm{wt} . \%$ and $30 \mathrm{GWd} / \mathrm{tU}, 4 \mathrm{wt} . \%$ and $45 \mathrm{GWd} / \mathrm{tU}, 5 \mathrm{wt} . \%$ and $60 \mathrm{GWd} / \mathrm{tU})$, which are three assemblies with PNAR Ratios of about 1.10, additional simulations were performed to calculate the PNAR Ratio for the case when no induced fission could take place. In other words, induced fission reactions became absorption reactions. This is a useful calculation as it indicates the signal expected if all the fissile and fertile isotopes were removed from the fuel. This change was accomplished by adding the "NONU" card to a re-simulation. The calculated PNAR Ratio for each of these assemblies with the NONU card is $1.001 \mathrm{H} / \mathrm{-}$ 0.002 . The propagated MCNP6 ${ }^{\mathrm{TM}}$ uncertainty for all PNAR Ratios is between 0.002 and 0.004 ; in other words, the vertical extent of each data point is approximately 3 
times the propagated statistical uncertainty calculated with $\mathrm{MCNP}^{\mathrm{TM}}$. The following are conclusions from Figure 4:

- The change in the PNAR Ratio is observed as a smoothly decreasing function of irradiation for a given initial enrichment.

- Fully irradiated assemblies, regardless of their initial enrichment, have nearly the same PNAR Ratio, 1.10 , in the case of VVER-440 assemblies in borated water. Almost all the assemblies to be measured at an encapsulation facility will be fully irradiated.

- There is a large difference in the PNAR Ratio between any irradiated assembly and a non-multiplying assembly; for the three assemblies simulated, the average difference in the PNAR Ratio is 0.137 .

In Figure 5 the PNAR Ratio is graphed as a function of the "net multiplication" as calculated by the MCNP6 $6^{\mathrm{TM}}$ code. Note that the net multiplication is calculated for the case of neutrons starting from all the pins in the assembly with the energy sampled from a Watt fission spectrum. The data points in Figure 5 include all the data points from Figure 4 as well as 6 additional data points. These additional data points are for the three fully irradiated assemblies for which the isotopic content was altered to represent the expected isotopic content after 40 and 80 -years of cooling. The 9 fully irradiated assemblies are clustered with PNAR Ratios around 1.10. From Figure 5 it is concluded that regardless of initial enrichment, cooling time or burnup, there is a smooth relationship between the PNAR Ratio and net multiplication, and, as in Figure 4, the large difference in the PNAR Ratio between any irradiated assembly and a nonmultiplying assembly is clearly observed.

\section{Location of Figures 5.}

\section{DYNAMIC RANGE AND UNCERTAINTY}

The conclusions drawn from the simulated data illustrated in Figure 4 and Figure 5 assume that the cumulative uncertainty inherent in a PNAR measurement is small enough such that the noted trends are not obscured. In the subsequent sections, the major anticipated uncertainties are analyzed to obtain an estimate of the expected aggregate uncertainty. The only uncertainty mentioned so far is the uncertainty of the $\mathrm{MCNP}^{\mathrm{TM}}$ simulation and that uncertainty has only involved the statistical uncertainty due to the number of particles run.

To gauge the importance of a given uncertainty, it is convenient to use the concept of dynamic range. For this study, we will primarily focus on the dynamic range between the following two cases: a fresh 4 wt. $\%$ assembly and a 4 wt.\%, $45 \mathrm{GWd} / \mathrm{tU}, 20$-year cooled assembly. For the data illustrated in Figure 4 and Figure 5, the PNAR Ratio for the 4 wt. $\%$ case changes from 1.216 to 1.105 for a dynamic range of $0.111+/-0.005$ for which the uncertainty is the propagated uncertainty of the MCNP6 ${ }^{\mathrm{TM}}$ simulation. 
Another useful uncertainty metric of comparison is the difference in the PNAR Ratio between a fully irradiated assembly and a non-multiplying assembly. In the $4 \mathrm{wt} . \%$ case, the PNAR Ratio changes from 1.105 to 1.002 for a change of $0.103+/-0.004$ for which the uncertainty is, once again, the propagated uncertainty of the MCNP6 ${ }^{\mathrm{TM}}$ simulation only. From these calculations, we see that the change in the PNAR Ratio as an assembly goes from fresh to fully irradiated is of the same magnitude as the change experienced between a fully irradiated assembly and a non-multiplying assembly.

From the facility operator of the pool where VVER-440 assemblies will be measured, we learned that the boron content of the water is expected to vary between $13 \mathrm{~g}$ and $15 \mathrm{~g}$ of boric acid per $\mathrm{kg}$ of water. For all the results simulated so far in this study 14 $\mathrm{g}$ of boric acid per $\mathrm{kg}$ of water was used. If the pool were maintained at $13 \mathrm{~g}$ of boric acid per $\mathrm{kg}$ of water, the simulated PNAR Ratio is $1.223+/-0.002$ for a fresh assembly and $1.111+/-0.003$ for a fully irradiated assembly; while if the pool were maintained at a $15 \mathrm{~g}$ of boric acid per $\mathrm{kg}$ of water, the simulated PNAR Ratio is 1.210 $+/-0.002$ for a fresh assembly and $1.103+/-0.002$ for a fully irradiated assembly. The change in PNAR Ratio for a change in boron content caused by the $2 \mathrm{~g}$ of boric acid per $\mathrm{kg}$ of water variation was 0.013 for a fresh assembly and 0.008 for a fully irradiated assembly. Hence, if a fully irradiated assembly were measured for a system calibrated at $14 \mathrm{~g}$ of boric acid per $\mathrm{kg}$ of water, the boron variation in the pool can be expected to create a 1-sigma uncertainty of 0.007 for a fresh assembly and 0.004 for a fully irradiated assembly if no correction in the measurement can be made for variation in the boron content of the water. The greater sensitivity of a fresh assembly to a change in the water boron content is expected because a fresh assembly is significantly more multiplying; hence, a given change in boron content will have a greater impact on changing the neutron multiplication.

For estimating an uncertainty in the PNAR Ratio due to the variation in the boron in the water for a Finnish implementation, we assume the following: (a) no correction can be made for variation in the boron content of the water, in other words measurements made of the boron content cannot be used, (b) the PNAR instrument is calibrated with a known boron content of $14 \mathrm{~g}$ of boric acid per $\mathrm{kg}$ of water, (c) the overwhelming majority of the assemblies to be measured are nearly fully irradiated; hence, the uncertainty is closer to that of a fully irradiated assembly. Given these assumptions and the simulations performed, a one-sigma uncertainty of 0.005 in the PNAR Ratio is suggested for the boron content variation when measuring VVER-440 assemblies. The decision to use 0.005 instead of 0.004 is a decision weighting the fact that most assemblies are fully irradiated but not all are. 
For all the simulation results presented in this report, the simulated assemblies were positioned in the center of the detector opening. The size of this opening was selected to match the storage rack dimensions used in Finland, where a $3.4 \mathrm{~mm}$ layer of water exists around the outside of the box that encases the assembly. When measuring actual assemblies, it is noted that they will be suspended from the end of a crane from which they are lowered into the detector and that they are likely to have varying degrees of irradiation-induced bending, resulting in a non-centered assembly position.

To assess the assembly positioning uncertainty once the instrument is built, multiple assemblies can be measured multiple times, while releasing the assembly from the crane and picking it up again between each measurement. To estimate the uncertainty from simulation before the instrument is built, we have simulated two displacement cases as well as the centered case and will compare among these cases. The two displacement cases are: (a) the assembly is positioned against one side of the detector wall with the assembly being centered along that side of the detector wall; (b) the assembly is pushed into one corner. For each of these cases, the PNAR Ratios and their uncertainties were calculated. [13]

Because the change in the PNAR Ratio is rather small given the detector opening anticipated for the Finnish deployment case, it was necessary to run significantly more particles relative to the simulations presented so far in this report. For this reason, a computer cluster at the Helsinki Institute of Physics was used in the simulations presented in this section. With this change of hardware came an associated change in software to MCNP5 V1.40 running with $0.60 \mathrm{c}$ [14] cross sections. To have confidence that the change of simulation code and cross sectional data did not impact the conclusions, the following points are noted: (a) All the assemblies depicted in Figure 4 and Figure 5 were simulated with MCNP5 and MCNP6 $^{\mathrm{TM}}$. Comparison between the two showed that there was a slight systematic shift between the two sets of codes and cross sections. Comparison between the two simulation cases showed that there was a systematic shift. Further research is needed to clearly identify the reason for the shift. It is the opinion of the authors that the difference in the available isotopic cross sections between the MCNP5 .60c and MCNP6 .80c cross is the major cause. (b) The results for which the MCNP5 code is being used is a comparison of MCNP5 results with MCNP5 results, a relative change. Specifically, we are comparing the PNAR Ratio calculated when the assembly is centered in the detector to the PNAR Ratio when the assembly is against the side or in the corner of the detector. Hence, it is expected that the differences responsible for the systematic shift between the two codes will cancel out.

Simulations "with the Cd-liner" and "without the Cd-liner" are performed for each of the 3 assembly locations (centered, against one side, corner) in the detector. It is thus possible to calculate 9 unique PNAR Ratios among these cases. For example, three 
PNAR Ratio values were calculated by combining the "with the Cd-liner" and centered assembly simulation with the "without Cd-liner" simulations for the assembly in the center, in the corner or against the side. Considering all permutations, the mean value is 1.1129 and the standard deviation from the mean is 0.0011 for 3 wt. $\%, 30 \mathrm{GWd} / \mathrm{tU} 20$-year cooled assembly. The uncertainty calculated for the MCNP5 statistics of each of the individual PNAR Ratios was also 0.0011 as well. An identical calculation made with a 4 wt.\%, $45 \mathrm{GWd} / \mathrm{tU} 20$-year cooled assembly produced the same mean value of 1.1129 and the standard deviation from the mean is 0.0014. The uncertainty calculated for the MCNP5 statistics of each of the individual PNAR Ratios was again 0.0011 . The two selected assembly cases were deemed to be the most representative, among the available cases, of the fuel assemblies to be measured in Finland.

The Monte Carlo statistical uncertainty on each individual point is comparable to the scatter among the 9 position combinations. The two sources of uncertainty can be added in quadrature to conclude that the one-sigma uncertainty of the PNAR Ratio resulting from positioning is not anticipated to be larger than 0.002 .

\section{UNCERTAINTY DUE TO COUNTING STATISTICS}

For a typical VVER assembly to be measured at the Finnish encapsulation facility a burnup of $\sim 32 \mathrm{GWd} / \mathrm{tU}$ and a cooling time of $\sim 40$ years is anticipated. The uncertainty due to counting statistics for such an assembly is expected to be 0.004 when both PNAR section measurements last 2 minutes. The uncertainty is expected to increase to 0.014 for a $17 \mathrm{GWd} / \mathrm{tU}, 60$ year cooled assembly if the count time is not increased. Yet, if the count time is increased for the particularly weak emitting assemblies or if more detector tubes are included, we expect that the one-sigma uncertainty due to counting statistics can be kept below 0.005 in the PNAR Ratio.

In the previous 3 sections, the one-sigma uncertainty in the PNAR Ratio was estimated for the variation in the boron content in the water, the positioning uncertainty of the assembly in the detector and the statistical uncertainty; values of $0.005,0.002$ and 0.005 were obtained, respectively. The quadrature sum estimated for the aggregate one-sigma uncertainty from these values is 0.008 .

It is interesting to note that the two largest uncertainties can reasonably be reduced by a factor of two in the following manner: (a) measure the boron content so that a correction to the PNAR Ratio calculation can be used, and (b) make the PNAR detector more efficient and/or count for longer. Yet, exactly what level of uncertainty is acceptable is an unresolved question, a question which this study will inform. If the two largest uncertainties are halved then a one-sigma uncertainty of 0.005 is possible. 
In the section on the simulated PNAR signal, we noted that the PNAR Ratio changed by 0.111 as a 4 wt.\% assembly was irradiated from fresh fuel to $45 \mathrm{GWd} / \mathrm{tU}$ and then we noted that the PNAR Ratio for this same assembly changed by another 0.106 units if the fissile material in the fuel was not allowed to undergo induced fission reactions. Now that we have a rough estimate of the PNAR instruments' uncertainty, we can see that the PNAR Ratio decreases by 14 sigma as the fuel is irradiated from fresh to fully irradiated and then the PNAR Ratio decreases by 13 sigma when a fully irradiated assembly is switched with a non-multiplying assembly. Hence, the neutron multiplication as measured by the PNAR Ratio changes by 1 sigma for each 3.2 $\mathrm{GWd} / \mathrm{tU}$ of burnup.

Both the estimation of a 13 sigma variation between a fully irradiated assembly and non-multiplying assembly, as well as the estimation that a 1 sigma variation in the PNAR Ratio corresponds to a $3.2 \mathrm{GWd} / \mathrm{tU}$ variation in the burnup inform the utility of the PNAR instrument. The following points are the reasons for including a PNAR instrument in the safeguards system: (1) PNAR indicates that fissile material is present in the assembly. (2) PNAR indicates that the assembly is multiplying at a level consistent with the declaration; the estimate that a one sigma variation in the PNAR Ratio corresponds to a $3.2 \mathrm{GWd} / \mathrm{tU}$ in burnup indicates that, within a parameter space that is a function of the initial enrichment and burnup, PNAR will be able to discern among assemblies and (3) PNAR provides the total neutron count rate with the ${ }^{3} \mathrm{He}$ tube and gross gamma intensity with a nitrogen filled ion chamber that must agree with the declaration.

Two suggestions are made with respect to how an inspectorate might use multiplication as a metric: (1) the calculations performed by the inspectorate, currently envisioned to be a SCALE $+\mathrm{MCNP}^{\mathrm{TM}}$ calculation, could simulate both the two parts of the PNAR measurement, with and without Cd present. Or, for a more rapid result, (2) a calibration factor could be determined between the measured PNAR Ratio and some already calculated quantity related to multiplication for each assembly such as a k-infinite effective value.

To this point in the paper we have focused on uncertainties that are associated with the PNAR hardware or the measurement environment: boron content of the water, counting statistics and assembly location in the detector. Yet, in the safeguards context, the measurements need to be connected to safeguards records recording the history of each assembly: assembly type, initial enrichment, burnup, cooling time, core shuffling patterns, etc. These records may be more or less detailed because the data required as part of a safeguards declaration are often less detailed than the records maintained by facilities. The more detailed the data provided in the declaration is, the greater the likelihood that a simulation based on the declaration will agree with measured values for a given assembly. An example related to this point is 
the use of average data vs. pin-by-pin data. Often pin-by-pin burnup data is available; yet, such detailed data does not need to be declared. However, the average burnup does need to be declared. A simulation using the pin-by-pin burnup is anticipated to results in more accurate simulations of the fuel, thus, closer agreement with the declaration. What level of detail is provided by the State to Euratom and the IAEA is outside of the scope of this work; the point being made here is that the State may want to provide more detail to increase the likelihood of agreement between the measured values and the values estimated from by simulations using the declared data as input.

The analytic technique expected to be used to connect the measurements to the declaration has been a subject of collaborative research for several years by Euratom and Oak Ridge National Laboratory researchers. [15, 16] Their research plan is to use Standardized Computer Analyses for Licensing Evaluation (SCALE) [17] to simulate the irradiation and cooldown of the fuel and $\mathrm{MCNP}^{\mathrm{TM}}$ to transport neutrons and gamma photons from the fuel to the detectors. The uncertainty inherent in the simulation of the PNAR Ratio and/or the net multiplication by the coupled SCALE and $\mathrm{MCNP}^{\mathrm{TM}}$ is a topic beyond the scope of the current research effort. The topic is noted here, as it will need to be addressed.

It merits noting that the data presented in this study are simulated, any uncertainty in the nuclear data or inaccuracies inherent in using simplistic geometric designs are present. Yet, it is worth noting that the significance of such uncertainties is reduced in the context of the PNAR technique by the fact that the PNAR Ratio is a ratio for which the numerator and the denominator are impacted similarly; thus, resulting in a minimal net variation in the PNAR Ratio. Experimental evidence of the utility of using PNAR with fresh $15 \times 15$ assemblies measured in water exists in publication [5].

\section{CONCLUSION}

By combining PNAR, PGET and a load cell, STUK has created an integrated NDA system that satisfies all the characteristics suggested by the NDA Focus Group convened by the IAEA as part of the ASTOR Experts Group. In this study, the performance of the PNAR instrument designed to measure VVER-440 fuel was examined. The PNAR instrument was included in the integrated system to measure the assembly's neutron multiplication; this capability is of particular interest in the context of the VVER-440 fuel in Finland because the instrument must work in a pool of borated water, which reduces the neutron multiplication. The uncertainty caused by variation in the boron content, assembly positioning in the detector and counting statistics were all examined to estimate an aggregate uncertainty of 0.008 in the PNAR Ratio. With this uncertainty, the anticipated sensitivity of the PNAR instrument for the VVER-440 case was quantified. To put this uncertainty in context, the substitution of a non-multiplying assembly for a fully irradiated assembly would result in a 13 sigma change in the PNAR Ratio. An additional manner of 
communicating this uncertainty, a 1 sigma variation in the PNAR Ratio corresponds to a change in the neutron multiplication that would be expected to be produced by an irradiation of $3.2 \mathrm{GWd} / \mathrm{tU}$; hence, the PNAR instrument can detect differences in neutron multiplication due to different degrees of irradiation. Although this study focused on the capability of the PNAR instrument, it merits emphasizing that in the Finnish encapsulation context, PNAR functions within an NDA system for which PGET has the role of detecting the localized emission of gamma rays from individual pins, PNAR has the role of verifying that fissile material is present, and SCALE + $\mathrm{MCNP}^{\mathrm{TM}}$ simulations have the role of verifying that the total neutron count rates, gross gamma intensity and multiplication are all consistent with the declaration.

\section{ACKNOWLEDGEMENTS}

The authors acknowledge the University of Helsinki and the Helsinki Institute of Physics for computing resources provided on the Alcyone computing cluster.

\section{REFERENCES}

[1] ASTOR Group Report 2011-2016, "Technologies Potentially Useful for Safeguarding Geological Repositories," International Atomic Energy Agency, STR384, Vienna, Austria 2017.

[2] T. Honkamaa, F. Levai, A. Turunen, R. Berndt, S. Vaccaro and P. Schwalbach, "A Prototype for Passive Gamma Emission Tomography," Symposium on International Safeguards: Linking Strategy, Implementation and People, Vienna, Austria, 2014.

[3] M. Mayorov, T. White, A. Lebrun, J. Brutscher, J. Keubler, A. Birnbaum, V. Ivanov, T. Honkamaa, P. Peura and J. Dahlberg, "Gamma Emission Tomography for the Inspection of Spent Nuclear Fuel," to be published in the IEEE NSS Conference Record, 2017.

[4] T. White, M. Mayorov, N. Deshmukh, E. Miller, L.E. Smith, J. Dahlberg and T. Honkamaa, "SPECT Reconstruction and Analysis for the Inspection of Spent Nuclear Fuel," to be published in the IEEE NSS Conference Record, 2017.

[5] D.M. Lee and L.O. Lindqvist, "Self-Interrogation of Spent Fuel," Los Alamos, LA-9494-MS, UC-15, 1982.

[6] J.L. Conlin, S.J. Tobin, J. Hu, T.H. Lee, H.O. Menlove, "Passive Neutron Albedo Reactivity with Fission Chambers," LA-UR-11-00521, 2010.

[7] A. Bolind, "Development of an Analytical Theory to Describe the PNAR and CIPN Nondestructive Assay Techniques," Annals of Nuclear Energy, vol. 66, pp. 167-176, 2014.

[8] T. Goorley et al., "Initial MCNP6 Release Overview," Nuclear Technology, vol. 180, pp. 298-315, Dec. 2012. 
[9] J.L. Conlin, D.K. Parsons, S.J. Gardiner, M.Gray, A.C. Kahler, M.B. Lee and M.C. White, "Continuous Energy Neutron Cross Section Data Tables Based upon ENDF/B-VII.1,” Los Alamos National Laboratory Report, LA-UR-13-20137, February, 2013.

[10] H.R. Trellue, M.L. Fensin, J.R. Richard, and J.L. Conlin, "Description of the Spent Nuclear Fuel Used in the Next Generation Safeguards Initiative to Determine Plutonium Mass in Spent Fuel," Los Alamos National Laboratory, LA-UR 11-00300, 2011.

[11] M.A. Humphrey, S.J. Tobin and K. Veal, "The Next Generation Safeguards Initiative's Spent Fuel Nondestructive Assay Project," Journal of Nuclear Material Management, vol. 3:XL, 2012.

[12] S.J. Tobin et al., "Experimental and Analytical Plans for the Non-Destructive Assay System of the Swedish Encapsulation and Repository Facilities," Symposium on International Safeguards: Linking Strategy, Implementation and People, Vienna, Austria, Vols. IAEA-CN-220-238, 2014.

[13] S.J. Tobin, P. Peura, T. Honkamaa' P. Dendooven, M. Moring, and C. BélangerChampagne, "Passive Neutron Albedo Reactivity in the Finnish Encapsulation Context," Finnish Radiation and Nuclear Safety Authority, ISBN 978-952-309-406-2, Helsinki Finland, December 2017.

[14] International Atomic Energy Agency, Nuclear Data Services, Vienna International Centre, P.O. Box 100, A-1400 Vienna, Austria, 2018.

[15] J. Hu, I.C. Gauld, J.E. Banfield and S.E. Skutnik, "Developing Spent Fuel Assemlby Standards for Advanced NDA Instrument Calibration - NGSI Spent Fuel Project," Oak Ridge National Laboratory Report, TM-2013/576, 2014.

[16] S. Vaccaro, J. Hu, J. Svedkauskaite, A. Smejkal, P. Schwalbach, P. De-Baere and I.C. Gauld, "A New Approach to Fork Measurements Data Analysis by RADARCRISP and ORIGEN Integration," IEEE Transactions on Nuclear Science, vol. 61, no. 4, pp. 2161-2168, March 2014.

[17] SCALE: "A Comprehensive Modeling and Simulation Suite for Nuclear Safety Analysis and Design," Oak Ridge National Laboratory, Oak Ridge, TN, ORNL/TM2005/39, June 2011. 
Fig. 1. Vertical (XZ plane) cross-sectional view of the VVER-440 PNAR detector along one side of a VVER-440 fuel assembly. Proportions are accurate.

Fig. 2. Horizontal (XY plane) cross-sectional view of the VVER-440 PNAR detector along one side of a VVER-440 fuel assembly. Proportions are accurate.

Fig. 3. Enlarged vertical (XZ plane) cross-section of Figure 1 indicating two VVER-440 PNAR detectors along two sides of a VVER-440 fuel assembly. Proportions are accurate.

Fig. 4. The VVER PNAR Ratio, simulated with borated water, is illustrated as a function of burnup for 12 assemblies of various initial enrichments and burnup values. The cooling time is 20 years. The vertical extent of each data point is approximately equal to 3-sigma of MCNP6 $^{\mathrm{TM}}$ statistical uncertainty.

Fig. 5. The VVER PNAR Ratio is graphed as a function of the net multiplication. The vertical extent of each data point is approximately equal to 3-sigma of $\mathrm{MCNP}^{\mathrm{TM}}$ statistical uncertainty. 
Figure 1

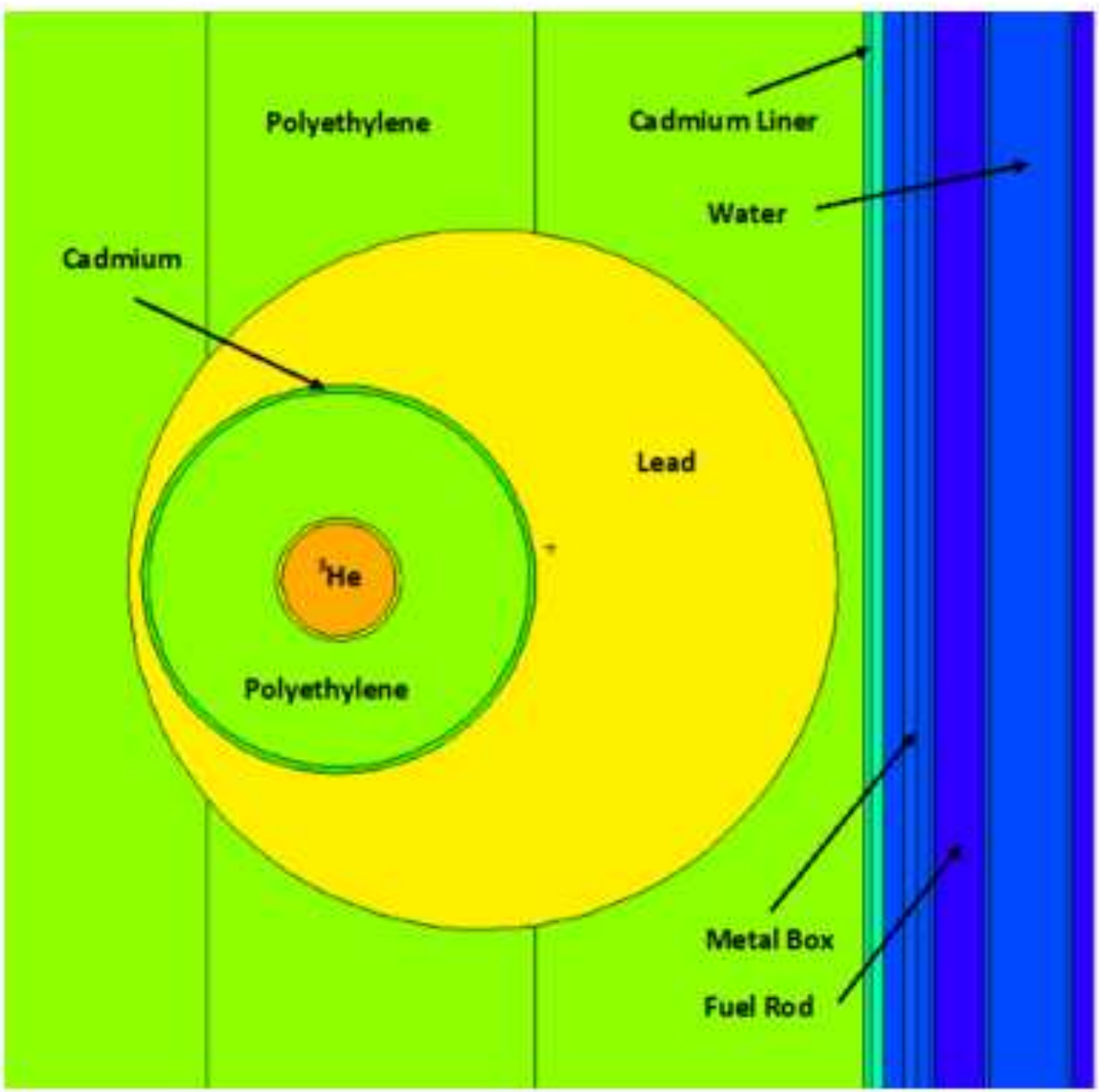




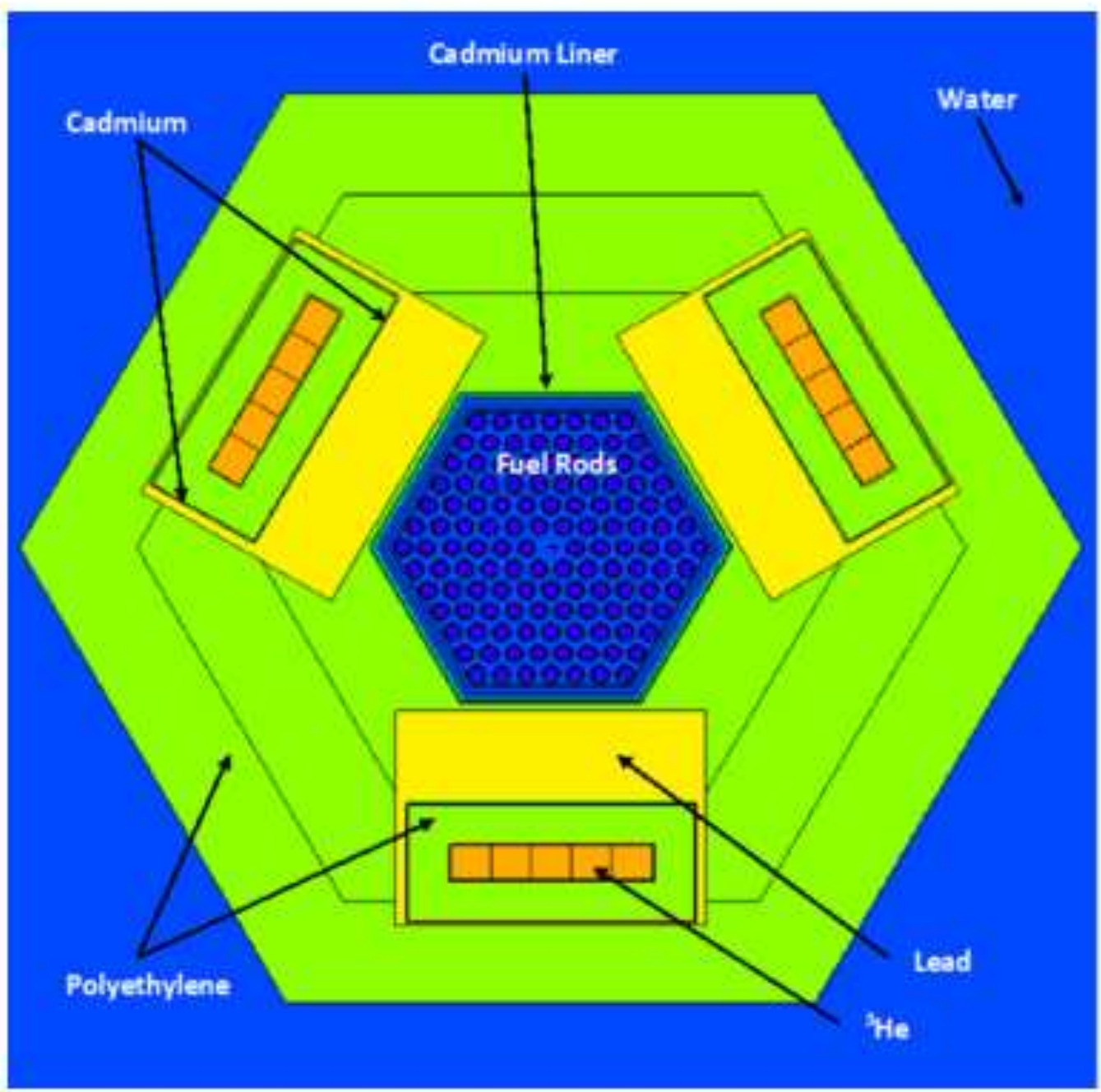


Figure 3

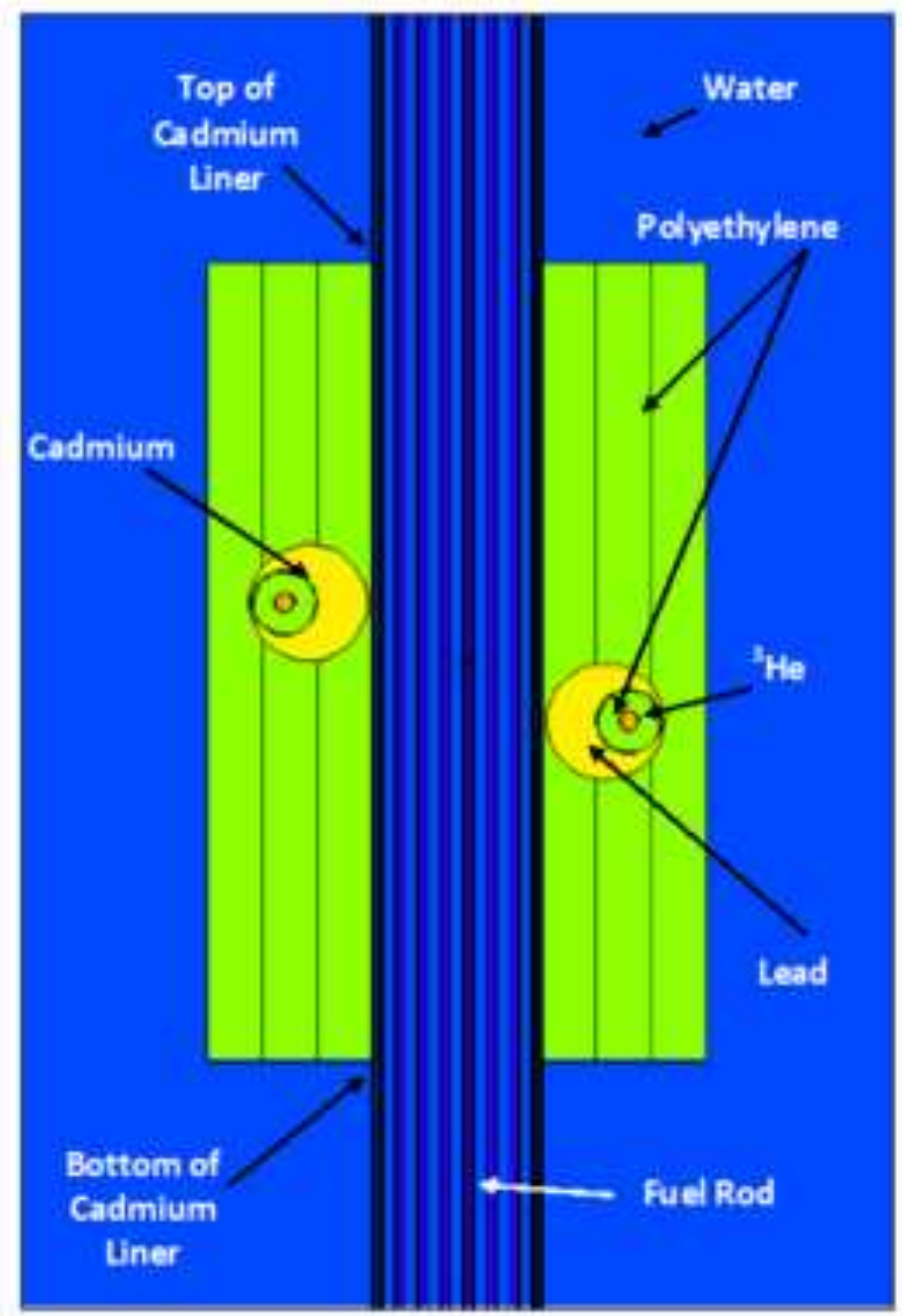




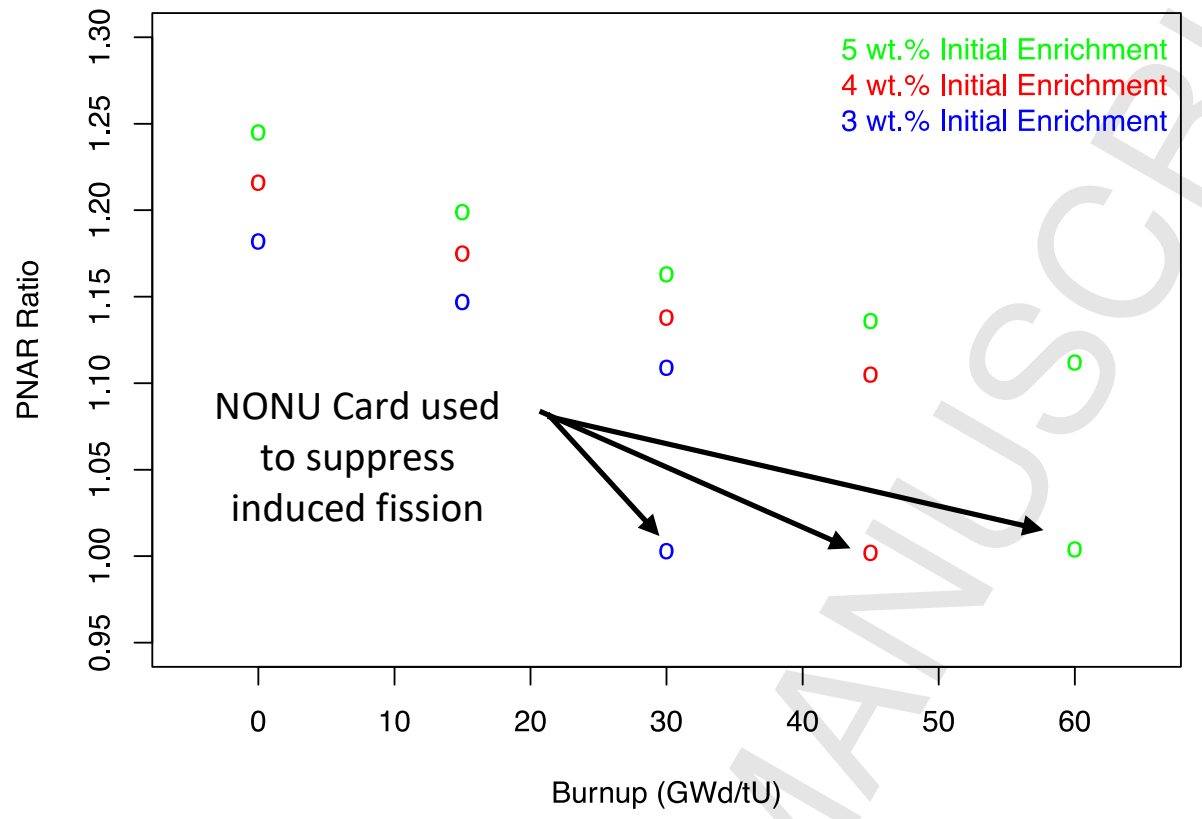




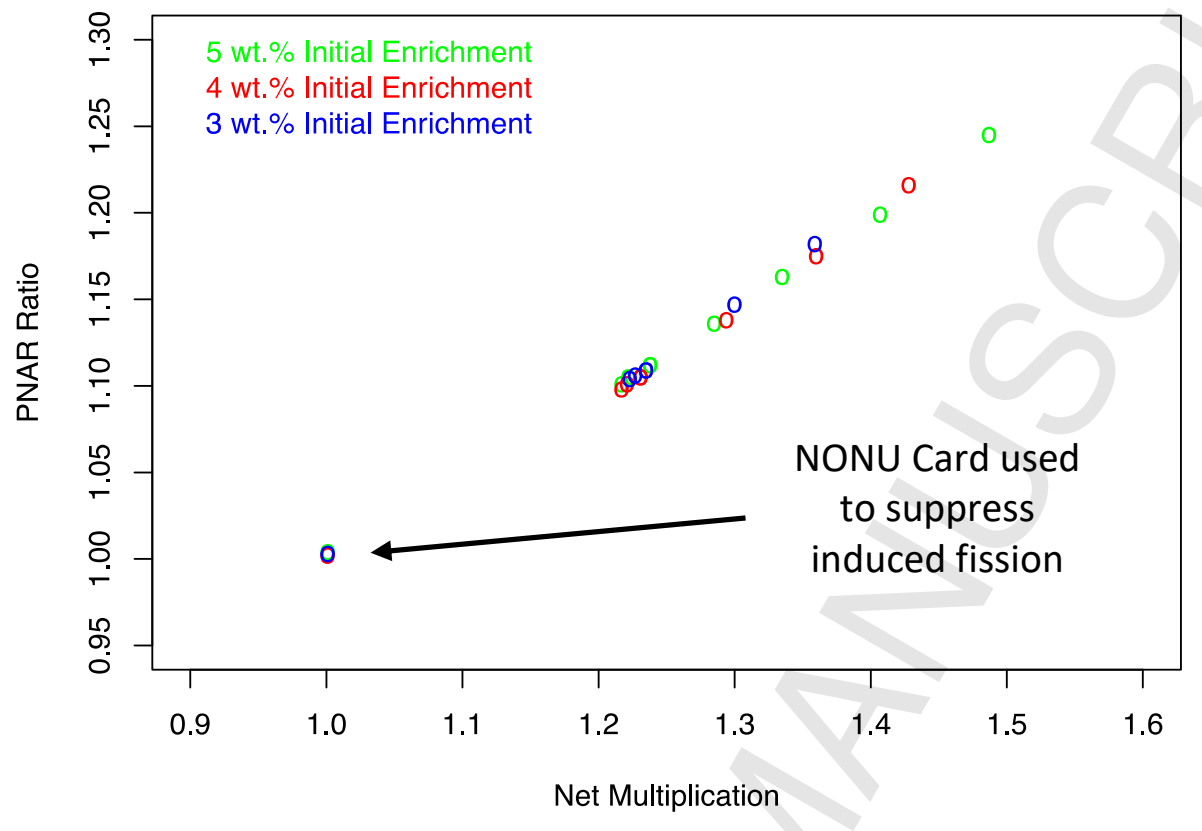

